\title{
Impacto da obesidade precoce na qualidade de vida e índices espirométricos de crianças e adolescentes
}

\author{
Impact of early obesity on quality of life and spirometric indices of children and \\ adolescents
}
Impacto de la obesidad temprana en la calidad de vida y los índices espirométricos en niños y adolescentes

Eloise Andrade Arruda ${ }^{1 *}$, Elivelton da Costa Fonseca ${ }^{2}$, Geraldo Roger Normando Junior ${ }^{3}$, Valéria Marques Ferreira Normando².

\begin{abstract}
RESUMO
Objetivo: Avaliar índices espirométricos e qualidade de vida de crianças e adolescentes com obesidade e sua correlação com o índice de massa corporal (IMC). Métodos: Estudo observacional, transversal, quantitativo, composto por 48 voluntários de ambos os sexos, idade de 8 a 12 anos, divididos em Grupo 1 (G1; eutróficos) e Grupo 2 (G2; obesos). Foram coletados dados antropométricos, espirométricos e a resposta dos questionários de qualidade de vida Pediatric Quality of Life Iventory (PedsQL ${ }^{\mathrm{TM}}$ ) child report e Pediatric Quality of Life Iventory (PedsQL ${ }^{\mathrm{TM}}$ ) parents report for child. Os dados estatísticos descritivos e inferenciais foram analisados com auxílio do software BioEstat versão 5.3 considerando $p \leq 0,05$. Resultados: A amostra, com média de idade de 10,42 $( \pm 1,28)$ anos, sendo $60,41 \%$ do sexo masculino, não apontou significância estatística para os domínios do questionário de QV entre G1 e G2, assim como para o perfil espirométrico. Porém, observou-se diferença significativa para os escores em quatro domínios quanto a percepção dos pais e dos seus filhos para o PedsQL4.0 Conclusão: Não houve correlação direta entre o IMC, dados espirométricos e a qualidade de vida dos voluntários estudados.
\end{abstract}

Palavras-chave: Obesidade infantil, Qualidade de vida, Função pulmonar.

\begin{abstract}
Objective: To evaluate spirometric indices and quality of life of children and adolescents with obesity and their correlation with body mass index (BMI). Methods: Observational, cross-sectional, quantitative study, comprising 48 volunteers of both sexes, aged 8 to 12 years, divided into Group 1 (G1; eutrophic) and Group 2 (G2; obese). Anthropometric, spirometric data and the response to the Pediatric Quality of Life Iventory (PedsQL ${ }^{\mathrm{TM}}$ ) $\mathrm{Child}^{\mathrm{Teport}}$ and Pediatric Quality of Life Iventory (PedsQL ${ }^{\mathrm{TM}}$ ) parents report for child questionnaires were collected. Descriptive and inferential statistical data were analyzed with the aid of the BioEstat software version 5.3 , considering $p \leq 0.05$. Results: The sample, with a mean age of $10.42( \pm 1.28)$ years, being $60.41 \%$ male, did not show statistical significance for the domains of the QOL questionnaire between G1 and G2, as well as for the spirometric profile. However, a significant difference was observed for scores in four domains regarding the perception of parents and their children for PedsQL4.0. Conclusion: There was no direct correlation between BMI, spirometric data and the quality of life of the studied volunteers.
\end{abstract}

Keywords: Childhood obesity, Quality of life, Lung function.

\section{RESUMEN}

Objetivo: evaluar los índices espirométricos y la calidad de vida de niños y adolescentes con obesidad y su correlación con el índice de masa corporal (IMC). Métodos: Estudio observacional, transversal, cuantitativo, compuesto por 48 voluntarios de ambos sexos, de 8 a 12 años, divididos en el Grupo 1 (G1; eutrófico) y el Grupo 2 (G2; obeso). Se recogieron datos antropométricos, espirométricos y la respuesta al informe infantil Pediátrico de la Calidad de Vida del Inventario (PedsQL ${ }^{\mathrm{TM}}$ ) y al informe Pediátrico de la Calidad de la Vida del Inventario (PedsQL ${ }^{\mathrm{TM}}$ ) de los padres para los cuestionarios infantiles. Los datos estadísticos descriptivos e inferenciales se analizaron con la ayuda del software BioEstat versión 5.3, considerando $p \leq 0.05$. Resultados: La muestra, con una edad media de $10,42( \pm 1,28)$ años, siendo el 60,41\% hombres, no mostró significación estadística para los dominios del cuestionario de CV entre G1 y G2, así como para el perfil espirométrico. Sin embargo, se observó una diferencia significativa para las puntuaciones en cuatro dominios con respecto a la percepción de los padres y sus hijos para PedsQL4.0. Conclusión: No hubo correlación directa entre el IMC, los datos espirométricos y la calidad de vida de los voluntarios estudiados.

Palabras clave: Obesidad infantil, Calidad de vida, Función pulmonar.

\footnotetext{
${ }^{1}$ Universidade de São Paulo (USP), São Paulo - SP, *E-mail: eloisea.arruda@gmail.com

2 Universidade do Estado do Pará (UEPA), Belém - PA.

3 Universidade Federal do Pará (UFPA), Belém - PA.
} 


\section{INTRODUÇÃO}

A Organização Mundial da Saúde (OMS), em 2014, fez um levantamento mostrando que 41 milhões de crianças menores que 5 anos estavam com sobrepeso ou obesidade em todo o mundo. Por meio de uma revisão sistemática com metanálise, identificou-se a prevalência de $14,1 \%$ de obesidade dentro do público infantil em território brasileiro (AIELO AM, et al., 2015).

Vários são os fatores que estão relacionados à obesidade infantil, principalmente em solo brasileiro, com destaque a região Norte, ou seja, em Rio Branco - AC, para crianças na faixa etária de até 5 anos, destacase os fatores socioeconômicos, local de domicílio na zona urbana e obesidade materna (SILVA DD, et al., 2019). O aumento na prevalência de obesidade na população infantil é preocupante, pois está associada a doenças cardiovasculares e ao desenvolvimento precoce de doenças crônicas da idade adulta, como: diabetes melito tipo II, hipertensão arterial sistêmica (HAS), dislipidemia, síndrome metabólica e eventos clínicos relacionados a aterosclerose - infarto agudo do miocárdio (IAM) e acidente vascular cerebral (AVC).

Ainda assim, há de se considerar três aspectos concernentes à ocorrência de doenças crônicodegenerativas devido a composição corporal excedente localizada no tronco, na região abdominal, assim como a gordura visceral, que insultam os fatores de risco (GUEDES ML, 2013; CAMARGOS ACR, et al., 2019).

Diante da análise dos fatores que compõem a vida dessa população com obesidade infantil, percebe-se que a Qualidade de Vida (QV), definida como uma percepção do próprio indivíduo em relação a sua vida em um contexto cultural e de sistemas de valores pré-existentes, e, também, em relação aos seus objetivos, expectativas, padrões e preocupações, estão afetadas, portanto, vários aspectos são utilizados para avaliar a QV, como: saúde, moradia, lazer, hábitos de atividade física e alimentação, criando, assim, uma ótica biopsicossocial para a QV das crianças (OMS, 1995).

O excesso de peso em crianças e adolescentes é um fator que impacta negativamente na QV desse público, principalmente no âmbito da saúde fisiológica e psicológica, levando, geralmente, essa criança a uma vida de inatividade e maus hábitos alimentares, além de sofrer estigmas nos ambientes sociais e escolares onde frequenta, logo, torna-se de extrema importância o rastreio de crianças e adolescentes com sobrepeso ou com obesidade, haja vista que a identificação precoce pode corroborar com desfechos positivos na vida adulta, evitando algumas doenças crônicas adquiridas e, com as medidas nutricionais corretas, promovendo um ganho na autoestima desse individuo (GOUVEIA MJ, et al., 2016; BACCHINI D, et al., 2017).

Ademais, a obesidade precoce pode ter repercussões negativas não só no sistema cardiovascular, mas também o sistema respiratório, como pode-se mostrar em uma revisão sistemática na qual identificaram que a obesidade pode desencadear, também, efeitos negativos nos volumes e na capacidades pulmonares de crianças e adolescentes, reduzindo, em especial, a Capacidade Residual Funcional (CRF) e consequentes Volume de Reserva Expiratório (VRE), Volume Residual (VR) e a Capacidade Pulmonar Total (CPT) (WINCK AD, et al., 2016). A presença de padrão restritivo em obesos foram referência conclusiva de outra revisão sistemática na comparação com indivíduos eutróficos, não só quanto a redução da CPT, mas também da capacidade vital forçada (CVF), e do volume expiratório forçado após um segundo (VEF1). Os autores reforçam a necessidade de instituir programas de assistência na promoção da qualidade de vida (MELO LC, et al., 2014).

Com a necessidade de avaliar com mais cuidado e abrangência do público infantil, questionários validados foram desenvolvidos com o objetivo de captar a percepção da criança e não apenas a obtenção do relato de seus pais e responsáveis. No Brasil foi validado o Questionário de Vida Relacionada a Saúde Pediátrica ou Pediatric Quality of Life Inventory (PedsQL), que integra abordagem genérica e doenças específicas (LOPES MT, et al., 2015).

Portanto, com base nas informações expostas, o objetivo deste estudo foi avaliar os índices espirométricos e a qualidade de vida de crianças e adolescentes com obesidade e sua correlação com o índice de massa corporal. 


\section{MÉTODOS}

Estudo observacional, transversal com abordagem quantitativa, realizado no município de Belém, no Estado do Pará, aprovado pelo Comitê de Ética em Pesquisa da Universidade da Amazônia (CEP) (protocolo $n^{\circ} 330.410$ ). Participaram do estudo 48 voluntários com idade entre 8 a 12 anos, selecionados por demanda espontânea, encaminhados por seus médicos para realização da espirometria.

Todos foram instruídos quanto aos objetivos e procedimentos que seriam realizados e, ao concordarem, assinaram o termo de consentimento livre e esclarecido (TCLE), assim como o termo de assentimento (TA). Foram incluídos e classificados conforme o Índice de Massa Corporal (IMC) e divididos em dois grupos:

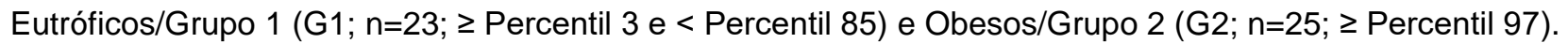

Foram incluídos no estudo voluntários cuja indicação clínica para a realização do teste espirométrico adveio da queixa de cansaço ao esforço físico sem histórico de doença cardiovascular ou pulmonar prévia. Inicialmente foram coletados o histórico médico/familiar, os dados pessoais e antropométricos, considerando o peso e a estatura como imprescindíveis a aferição antes da realização da espirometria, por meio de balança digital com precisão de $100 \mathrm{~g}$ e o estadiômetro com precisão de $1 \mathrm{~mm}$. Todos os dados foram registrados e armazenados em planilha eletrônica.

Antes da realização do teste o voluntário permaneceu em repouso de cinco a dez minutos para que dessa forma nenhum esforço físico pudesse interferir no teste, e, além disso, recebeu orientação previa para esclarecimento de todo o andamento do exame.

Durante o exame, o voluntário se manteve na posição sentada, cabeça em posição neutra e com pinça nasal, com os lábios bem cerrados em volta do bocal para evitar o escape de ar. Para a técnica, o voluntário realizou em duas etapas diferentes, ou seja, para a manobra lenta ocorreu tanto a inspiração quanto a expiração de forma lenta e profunda, e para a manobra forçada a inspiração máxima foi seguida da expiração forçada e sustentada, por seis segundos em média.

O voluntário realizou no mínimo três curvas aceitáveis e reprodutíveis, para a escolha da melhor curva. Os que realizaram o procedimento incorretamente após seis tentativas, foram descartados da amostra (JOHNS DP, et al., 2015). Ressalta-se que o laudo dos exames foi conduzido às cegas de forma que não houvesse qualquer tendência inferencial na leitura dos dados quantitativos. As variáveis espirométricas avaliadas em foco neste estudo foram: capacidade vital lenta (CVL), capacidade vital forçada (CVF), volume expiratório forçado no primeiro segundo (VEF1) e a relação VEF1/CVF (Índice de Tiffeneau), por meio do espirômetro a volume SPIROM2 associado ao software Spiromatic versão 2.0.

Para tal, foram utilizadas as manobras, lenta e forçada para o alcance dos volumes e fluxos expiratórios. Os valores obtidos foram comparados a valores previstos adequados para a população avaliada, considerando os valores de normalidade recomendados pelas Diretrizes para Testes de Função Pulmonar da Sociedade Brasileira de Pneumologia e Tisiologia (2002).

$\mathrm{Na}$ obtenção de exito do voluntário quanto a reprodutibilidade e aceitabilidade na realização do teste, o examinador entregava os dois questionários de Qualidade de Vida para preenchimento, sendo o Pediatric Quality of Life Iventory (PedsQL ${ }^{\mathrm{TM}}$ ) children report para os voluntários e Pediatric Quality of Life Iventory (PedsQL ${ }^{\mathrm{TM}}$ ) parents report for child para os pais ou responsáveis pelos mesmos, na versão 4.0-PortugêsBrasil. Os preenchimentos ocorreram sem houvesse interferência do examinador/pesquisador.

O referido questionário caracteriza-se por condicionar uma autoavaliação na visão da criança/adolescentes e a percepção dos pais quanto a qualidade de vida do filho. A versão do instrumento a partir de 8 anos contempla 23 itens divididos quatro domínios: físico (8 itens), emocional (5 itens), social (5 itens) e escolar (5 itens).

Os escores totais, Psicossocial (PS) e Saúde Geral (SG) foram computados pela soma dos itens dividida pelo número de itens respondidos. Os valores foram operacionalizados numa escala linear inversa de 0 100 , onde quanto maior a pontuação melhor a qualidade de vida relacionada à saúde. Cabe ressaltar que houve a atenção dos pesquisadores em solicitar a permissão da Mapi Research Trust, responsável pelos direitos autorais dos referidos questionários antes de serem aplicados. 
Para análise estatística foi utilizado o programa BioEstat versão 5.3, com a aplicação do teste ShapiroWilk a fim de analisar os dados em relação à distribuição da curva de normalidade. Para caracterização da amostra e distribuição dos dados foi utilizada a estatística descritiva, por meio de medidas de tendência central e dispersão. Os dados obtidos foram expressos em média+DP para as variáveis paramétricas. A análise estatística foi realizada por meio de métodos estatísticos descritivos e inferenciais, apresentados por distribuição de frequências absolutas e relativas. Para análise entre os grupos $\mathrm{G} 1$ e G2, utilizou-se o teste $t$ de Student para amostras independentes e variáveis paramétricas e o teste de Mann-Whitney para variáveis não-paramétricas, considerando o nível de significância de $5 \%(p<0,05)$.

\section{RESULTADOS}

A média de idade da amostra foi de $10,42(+1,28)$ anos, sendo $60,41 \%$ do sexo masculino. A prevalência entre os sexos foi similar no G1 (47,82\% feminino e $52,17 \%$ masculino), porém no G2 houve maior prevalência do sexo masculino (68\%). A distribuição dos voluntários, conforme o IMC, os dados obtidos nos questionários de QV e Espirometria foram descritos na Tabela 1, a qual aponta homogeneidade da amostra e aponta que não houve diferença estatística significante em todos os domínios do questionário de QV PedsQL4.0 entre os grupos, quando comparado a percepção dos voluntários, o mesmo foi observado em relação a percepção dos pais.

Tabela 1 - Médias e desvio padrão das variáveis antropométricas, perfil espirométrico e qualidade de vida dos voluntários (8-12 anos), 2014.

\begin{tabular}{|c|c|c|c|}
\hline \multirow{2}{*}{\multicolumn{3}{|c|}{$\begin{array}{l}\text { Variáveis } \\
\text { Sexo }\end{array}$}} & \multirow{10}{*}{ P - Valor } \\
\hline & & & \\
\hline Masculino & 12 & 17 & \\
\hline Feminino & 11 & 8 & \\
\hline Idade (anos) & $10,59+1,26$ & $10,28+1,31$ & \\
\hline Altura (m) & $1,47+0,11$ & $1,49+0,07$ & \\
\hline Peso (Kg) & $37,7+8,21$ & $54,98+13,07$ & \\
\hline IMC & $17,28+1,71$ & $24,52+3,74$ & \\
\hline Percentil & $54,09+26,67$ & $97,48+1,76$ & \\
\hline $\begin{array}{l}\text { Qualidade de vida - PedsQL }{ }^{\mathrm{TM}} \\
\text { childreport }\end{array}$ & $\mathrm{MD}+\mathrm{DP}[\mathrm{T} \%]$ & MD + DP [T\%] & \\
\hline Físico & $70,29+16,76[70,29]$ & $78,24+14,34[78,24]$ & $p=0.1909$ \\
\hline Emocional & $63,47+16,19[63,47]$ & $68,59+20,65[68,59]$ & $\mathrm{p}=0.2195$ \\
\hline Social & $80+22,46[80]$ & $77,57+17,90[77,57]$ & $\mathrm{p}=0.6722$ \\
\hline Escolar & $67,82+16,50[67,82]$ & $67,91+24,11[67,91]$ & $\mathrm{p}=0.8911$ \\
\hline PS & $214,3:+45,26[71,45]$ & $214,08+48,44[71,67]$ & $\mathrm{p}=0.8044$ \\
\hline SG & $284,64+56,98[71,16]$ & $292,33+60,67[72,86]$ & $\mathrm{p}=0.4765$ \\
\hline $\begin{array}{l}\text { Qualidade de vida - PedsQL }{ }^{\mathrm{TM}} \\
\text { parentreport }\end{array}$ & MD + DP [T\%] & $\mathrm{MD}+\mathrm{DP}[\mathrm{T} \%]$ & \\
\hline Físico & $65,97+24,64[65,97]$ & $68,13+15,77[68,13]$ & $p=0.74477$ \\
\hline Emocional & $64,78+24,65[64,78]$ & $64,22+22,83[64,22]$ & $p=0.7948$ \\
\hline Social & $75+21,05[75]$ & $62,89+25,66[62,89]$ & $\mathrm{p}=0.1222$ \\
\hline Escolar & $55,65+24,41[55,65]$ & $49,22+24,62[49,22]$ & $\mathrm{p}=0.3018$ \\
\hline PS & $195,43+60,09[65,14]$ & $176,32+51,08[59,95]$ & $\mathrm{p}=0.5845$ \\
\hline SG & $261,40+81,66[65,35]$ & $255,90+42,32[63,78]$ & $\mathrm{p}=0.9835$ \\
\hline Espirometria & $M D+D P$ & $M D+D P$ & \\
\hline CVL & $2.25+0.44$ & $2.47+0.59$ & $\mathrm{p}=0.3119$ \\
\hline CVF & $2,38+0,45$ & $2,62+0,63$ & $\mathrm{p}=0.2081$ \\
\hline VEF1 & $2,02+0,37$ & $2,16+0,52$ & $\mathrm{p}=0.4390$ \\
\hline VEF1/CVF & $85,55+8,35$ & $82,83+7,91$ & $\mathrm{p}=0.2531$ \\
\hline
\end{tabular}

Legenda: IMC: Índice de massa corporal; F: feminino; M: masculino; MD: Média; DP: Desvio Padrão; PS: Psicossocial; SG: Saúde Geral; CVL: Capacidade Vital Lenta; CVF: Capacidade Vital Forçada; VEF1: Volume Expiratório Forçado no primeiro segundo; VEF1/CVF: relação do VEF1 com a CVF ou índice de Tiffeneau. Fonte: Arruda EA, et al., 2014. 
Quando se compara a percepção dos pais com a dos seus filhos em relação a QV (Tabela 2), individualmente, observou-se que no G1 não houve diferença significativa entre os domínios, porém para o G2 observa-se significância para quatro domínios, ou seja, social $(p=0.0157)$, escolar $(p=0.0039)$, psicossocial $(p=0.0030)$ e da saúde geral $(p=0.0055)$.

Tabela 2 - Comparação entre as médias obtidas nos questionários PedsQL ${ }^{\mathrm{TM}}$ childreport e parentreport para cada grupo.

\begin{tabular}{ccc}
\hline Domínios & $\begin{array}{c}\text { G1 } \\
(\mathbf{n}=\mathbf{2 3})\end{array}$ & $\begin{array}{c}\text { G2 } \\
(\mathbf{n}=\mathbf{2 5})\end{array}$ \\
\hline Físico & $\mathrm{p}=0.4902$ & $\mathrm{p}=0.0523$ \\
Emocional & $\mathrm{p}=0.8330$ & $\mathrm{p}=0.9536$ \\
Social & $\mathrm{p}=0.4290$ & $\mathrm{p}=0.0157^{*}$ \\
Escolar & $\mathrm{p}=0.0538$ & $\mathrm{p}=0.0039^{*}$ \\
PS & $\mathrm{p}=0.2343$ & $\mathrm{p}=0.0030^{*}$ \\
SG & $\mathrm{p}=0.3229$ & $\mathrm{p}=0.0055^{*}$ \\
\hline
\end{tabular}

Legenda: PS: Psicossocial; SG: Saúde Geral. " $p<0$,05. Fonte: Arruda EA, et al., 2014.

\section{DISCUSSÃO}

A Qualidade de Vida (QV) sempre foi discutida na literatura com vários questionários específicos para o público adulto, porém, devido as particularidades do público infantil, foi necessário a criação do Peds QL $^{\mathrm{TM}}$ em 1987, com sua tradução transcultural para o português em 2008. O Peds $\mathrm{QL}^{\mathrm{TM}}$ é uma versão genérica que avalia a QV de qualquer criança e adolescente de 2 a 18 anos de idade, ou seja, são aquelas crianças saudáveis, sem nenhuma condição de doença específica, e que hoje, com vários acréscimos feitos ao longo do tempo, se encontra na sua versão 4.0, podendo ter a percepção das crianças (chlidren report) e a percepção dos pais (parents report for child) (VARNI JW, et al., 2001; KLATCHOIAN DA, et al., 2008).

Lima RD, et al. (2019) analisaram os dados antropométricos baseados na medição do percentual e gordura da prega cutânea do tríceps de crianças de 7 a 10 anos e descobriram que, primeiro, esse método possui uma positiva relação com a taxa de Índice de Massa Corporal (IMC) com concordância satisfatória entre os métodos; segundo, o estudo mostrou que a maior porcentagem de crianças acima do peso estava dentro do sexo masculino, fato que corrobora com os achados do estudo presentes na Tabela 1. Com esses resultados pode-se inferir que o IMC não teve influência na QV da amostra estudada, tanto no que se refere a percepção do voluntário, quanto na percepção do responsável sobre a QV.

Achados que compararam os efeitos de um tratamento multidisciplinar com e sem o acompanhamento psicológico de adolescentes obesos do Estado de Pernambuco, apontaram como resultados que $100 \%$ dos participantes que concluíram o tratamento apresentou algum tipo de queda na QV, a qual foi avaliada também pelo Peds $\mathrm{QL}^{\mathrm{TM}}$, principalmente nos tópicos como aceitação social e automotivação; ademais, ainda atestam que a presença do psicólogo contribui positivamente para uma melhora na QV desse público (FREITAS CRM, et al., 2016).

Delgado-Floody $\mathrm{P}$, et al. (2019), encontraram em um estudo realizado com 419 adolescentes chilenos com idade entre 14 e 17 anos mostrou que o desempenho escolar não tem relação com o estado nutricional dos participantes, dados estes que corroboram com os achados desta pesquisa, pois também não houve diferença significativa no domínio escolar nos G1 e G2, como podem ser vistos nos dados presentes na Tabela 1.

Observou-se que os obesos obtiveram média superior aos eutróficos em quase todos os domínios do questionário. Já no relato dos pais, os obesos superaram as médias apenas no domínio físico, estando abaixo nos demais. Tais dados do relato dos pais entram em discordância com os resultados de uma revisão sistemática, as quais fizeram um levantamento dos dados da relação entre a obesidade infantil e os aspectos psicossociais na visão das crianças obesas e concluíram que nem todas experimentam problemas psicossociais e ainda alertam sobre as generalizações feitas nessa população (SAGAR R e GUPTA T, 2018). 
Farias ES, et al. (2019), observaram em estudo no qual associavam a queda no desempenho escolar tanto pelo excesso de peso, quanto pela alta taxa de inatividade física de adolescentes de Porto Velho, sudoeste da Amazônia brasileira, acusando o impacto na percepção emocional.

Rankin J, et al. (2016) por meio de uma revisão sistemática sobre as consequências psicológicas, psiquiátricas e psicossomáticas da obesidade infantil, afirmaram que crianças obesas possuem uma autoestima muito prejudicada quando comparadas aquelas dentro da faixa de peso normal, tais dados que corroboram, também, com os achados da pesquisa, onde os domínios que sofreram maior impacto na percepção dos voluntários no G2 foram o emocional e o escolar.

No que diz respeito a percepção dos pais observou-se médias $<70 \%$ em todos os domínios nos dois grupos, exceto o domínio social do G1, com 75\%. Os domínios de maior impacto foram o escolar e 0 psicossocial, com médias < 60\%. Ruiter ELM, et al. (2020) apontaram resultados que entram em discordância com os achados do estudo em pauta, uma vez que atestaram uma subestimação de pais holandeses em relação a situação de obesidade dos filhos, fato que repercute em todos os âmbitos da vida da criança, além de prejudicar o rastreio e a intervenção precoce por parte dos profissionais de saúde contra a obesidade infantil.

Nascimento NNR, et al. (2016) fizeram um estudo que corroboram com os dados da pesquisa, no qual eles avaliaram, na perspectiva dos pais e cuidadores, a Qualidade de Vida Relacionada à Saúde (QVRS) de crianças e adolescentes com sobrepeso e obesidade de Uberlândia - MG e tiveram como resultados que os pais possuem uma visão da QVRS de seus filhos muito frágil, principalmente em relação ao ambiente escolar, onde eles afirmam que seus filhos possuem poucos amigos e sofrem muito preconceito por conta do excesso de peso.

Já no G2 houve diferença significativa nas médias obtidas em quase todos os domínios, também inferior, exceto o emocional, resultado pode indicar uma dificuldade de relacionamento entre pais e filhos ou uma percepção pessimista dos pais sobre a QV do filho devido a condição física.

Xanthopoulos MS, et al. (2017), apontaram dados que entram em consonância com a pesquisa, uma vez que ao avaliarem a QV por meio do PEDS QLTM, na percepção dos pais e cuidadores, de crianças norte americanas obesas com e sem Síndrome de Down (SD) concluíram que ambos os grupos obtiveram médias em que indicaram uma baixa QV na visão dos seus responsáveis, exceto, também, do domínio emocional, com um agravante para o grupo das crianças com SD, que obtiveram médias extremamente baixas.

Em relação a função pulmonar observou-se quatro perfis espirométricos: normal, redução isolada de Volume Expiratório Forçado no primeiro segundo (VEF1), distúrbio ventilatório obstrutivo (DVO) leve e distúrbio ventilatório restritivo (DVR) leve, classificados conforme o Consenso Brasileiro de Espirometria (CBE).

Andrade FSSD, et al. (2017), verificaram se o fator sexo, idade e IMC influenciariam na função pulmonar, por meio do Pico de Fluxo Expiratório, de crianças entre 8 e 10 anos e puderam inferir que nenhum desses fatores supracitados possuem influencia numa piora dos escores relativos a função pulmonar, haja vista que estes se apresentaram dentro da normalidade, achados que se tornam discordantes em relação ao estudo.

Os laudos espirométricos no G1 apontaram normalidade em 60,87\%, 34,78\% DVO leve e 4,35\% de DVR leve, sendo que no G2 48\% normais, $48 \%$ DVO leve e $4 \%$ de redução isolada de VEF1. Não houve diferença significativa ao comparar o percentual predito (T\%) das variáveis estudadas entre os dois grupos, o que indica que, para a população estudada, o IMC não interferiu diretamente no desempenho pulmonar, porém observou-se que no G2 houve maior prevalência de padrões obstrutivos, que podem estar relacionados a obesidade.

Lang JE (2014) comentou em seu estudo que, apesar de não haver uma associação clara entre a obesidade e os parâmetros de função pulmonar em crianças pré-púberes, a obesidade pode estar associada a obstrução de vias aéreas no sexo masculino. Arias-Rico JA, et al. (2016) apontou resultados de que o IMC ou a Gordura Corporal de crianças mexicanas entre 5 e 11 anos não tem influência direta na espirometria. 
Na aplicação do teste de correlação entre o perfil espirométrico e os domínios PS e SG, não houve significância estatística. Assunção SNF, et al. (2014), registram resultados similares uma vez que não observou correlação entre parâmetros antropométricos em relação aos distúrbios respiratórios, exceto para VEF1/CVF em indivíduos com DVM (Distúrbio ventilatório misto). Todavia, Kochli S, et al. (2019) realizou um estudo que teve como premissa investigar a associação entre a obesidade infantil e a aptidão cardiorrespiratória e teve como resultados que a diminuição da relação VEF1/CVF ocorre com mais significância com o aumento do IMC, discordando, então, dos dados achados deste presente estudo.

\section{CONCLUSÃO}

O aumento do IMC não interferiu significativamente na qualidade de vida dos participantes deste estudo, haja vista que seus valores no questionário PEDS QL ${ }^{\mathrm{TM}}$ não apontaram diferença estatística relevante; além disso, não houve correlação direta entre perfil espirométrico e a população estudada, pois os valores obtidos na espirometria pelo grupo G1 e pelo grupo G2 não apresentaram disparidade significante. Contudo, seu potencial ainda não foi inteiramente explorado, sendo assim sugere-se a continuidade do estudo com a inserção diagnóstica sobre os hábitos social e nutricional, assim como com um número maior de indivíduos para que seus efeitos mediante diferentes gêneros e faixa etária possam servir como complemento a leitura do perfil metabólico e o acompanhamento longitudinal dos dados, pois partindo dessas novas premissas a pesquisa pode ser mais precisa quanto aos resultados, gerando um melhor retorno possível para a toda a comunidade científica.

\section{REFERÊNCIAS}

1. AIELLO AM, et al. Prevalence of obesity in children and adolescents in Brazil: a meta-analysis of cross-sectional studies. Current Pediatric Reviews, 2015; 11(1): 36-42.

2. ANDRADE FSSD, et al. Lung function and functional capacity in school age children. Fisioter Mov. 2017 Jan/Mar;30(1):77-84.

3. ARISAS-RICO J, et al. Obesidad infantil y su relación con indicadores cardiopulmonares en escolares mexicanos. Aquichan, 2016; 16(2): 148-158.

4. ASSUNÇÃO SNF, et al. Função pulmonar de crianças e adolescentes sem sintomas respiratórios e excesso de peso. Jornal Brasileiro de Pneumologia, 2014; 40(2): 134-141.

5. BACCHINI D, et al. The interplay among BMI Z-score, peer victimization, and self-concept in outpatient children and adolescents with overweight or obesity. Jornal of Childhood Obesity, 2017; 13: 242-249.

6. CAMARGOS ACR, et al. Prevalência de sobrepeso e de obesidade no primeiro ano de vida nas Estratégias Saúde da Família. Cad. Saúde Colet., 2019, Rio de Janeiro, 27 (1): 32-38.

7. DELGADO-FLOYD $P$, et al. Calidad de vida, autoestima, condicion física y estado nutricional en adolescentes y su relación con el rendimiento académico. Revista de la Sociedad Latinoamericana de Nutrición, 2019; 69(3): $174-181$.

8. FARIAS ES, et al. Comportamento inativo em estudantes adolescentes da Amazônia ocidental brasileira. Revista Paulista de Pediatria, 2019; 37(3): 345-350.

9. FREITAS CRM, et al. Effects of a psychological intervention on the quality of life of obese adolescents under a multidisciplinary tratament. Journal of Pediatric, 2017; 93(2): 185-191.

10. GOUVEIA MJ, et al. Imagem corporal e qualidade de vida na obesidade pediátrica. Psicologia, Saúde e Doença, 2016; 17: 52-59.

11. GUEDES ML. Avaliação da qualidade de vida em crianças e adolescentes com asma. Dissertação (Mestrado em Medicina) - Instituto Ciências da Saúde. Universidade da Beira Interior, Covilhã, 2013; 55 p.

12. JOHNS, D.P. et al. Spirometer Users' and Buyers' Guide - Revised 2015. Melbourne: Nat. Asthma Council, 2015.

13. KLATCHOIAN DA, et al. Quality of life of children and adolescents from São Paulo: reliability and validity of the Brazilian version of the Pediatric Quality of Life Inventory ${ }^{\mathrm{TM}}$ version 4.0. Journal of Pediatric, 2008; 84: 800-812.

14. KOCHIL S, et al. Lung function, obesity and physical fitness in young children: the examin youth study. Journal Respiratory Medicine, 2019; 159: 1-28.

15. LANG GE. Exercise, obesity and asthma in children and adolescents. The Journal of Pediatrics, 2014; 90(3): 215217.

16. LIMA RD, et al. Ocorrência de excesso de peso em escolares e análise da concordância entre métodos antropométricos. Revista Brasileira de Cineantropometria e Desempenho Humano, 2020; 22: 1-9.

17. LOPES MT, et al. Confiabilidade da tradução da versão brasileira do questionário PedsQL - DREA para avaliação da qualidade de vida de crianças e adolescentes. Jornal Brasileiro de Nefrologia, 2015; 37(2): 158-165.

18. MELO LM, et al. Obesidade e função pulmonar: uma revisão sistemática. Einstein. 2014;12(1):120-5.

19. NASCIMENTO MMR, et al. Parent's perception of health-related quality of life in children and adolescents with excess weight. Journal of Pediatric, 2016; 92: 65-72.

20. ORGANIZAÇÃO MUNDIAL DA SAÚDE. 2014. Estatísticas de saúde mundial. Disponível em: https://apps.who.int/iris/bitstream/handle/10665/112738/9789240692671_eng.pdf;jsessionid=CA9AD110444531394 540E3137B1CA4A7? sequence=1. Acesso em: 26 jun. 2020. 
21. ORGANIZAÇÃO MUNDIAL DA SAÚDE. 1995. Estado físico: uso e interpretação da antropometria. Disponível em: https://www.who.int/childgrowth/publications/physical_status/en/ . Acesso em: 26 jun. 2020.

22. RANKIN J, et al. Psychological consequences of childhood obesity: psychiatric comorbity and prevention; Journal Adolescent Health, Medicine and Therapeutics, 2016; 6(7): 125-146.

23. RUITER ELM, et al. Parent's underestimation of their child's weight status, moderating factors and change over time: a cross-sectional study. Journal Plos One, 2020; 15(1): 1-16.

24. SAGAR R, GUPTA T. Psychological aspects of obesity in children and adolescests. Journal Indiano of Pediatric, 2018; 85(7): 554-559.

25. SILVA DD, et al. Prevalência e fatores associados à obesidade em crianças menores que cinco anos no município de Rio Branco - Acre. Journal of Human Growth and Development, 2019; 29(2): 263-273.

26. Sociedade Brasileira de Pneumologia e Tisiologia. Diretrizes para Testes de Função Pulmonar. J Bras Pneumol. 2002;28(Suppl 3):S1- S82.

27. VARNI JW, et al. PedsQL 4.0: reability and validity of the pediatric quality of life inventory version 4.0 generic core scales in healthy and patient populations. Journal Medical Care, 2001; 39: 800-812.

28. WINCK AD, et al. Efeitos da obesidade sobre os volumes e capacidades pulmonares em crianças e adolescentes: uma revisão sistemática. Revista Paulista de Pediatria, 2016; 34(4): 510-517.

29. XANTHOPOULOS MS, et al. Qualidade de vida relatada pelo cuidador em jovens com Síndrome de Down. Tele Jornal de Pediatria, 2017; 1-7. 